\title{
Generic Sandpile Models Have Directed Percolation Exponents
}

\author{
P. K. Mohanty and Deepak Dhar \\ Tata Institute of Fundamental Research, Homi Bhabha Road, Mumbai-400 005, INDIA
}

\begin{abstract}
We study sandpile models with stochastic toppling rules and having sticky grains so that with a non-zero probability no toppling occurs, even if the local height of pile exceeds the threshold value. Dissipation is introduced by adding a small probability of particle loss at each toppling. Generically, for models with a preferred direction, the avalanche exponents are those of critical directed percolation clusters. For undirected models, avalanche exponents are those of directed percolation clusters in one higher dimension.

PACS numbers : 05.65.+b, 45.70.Ht, 64.60.Ak, 64.60.Fr
\end{abstract}

In recent years, there has been a lot of interest in the study sandpile models as models of real granular matter [1], and also as paradigms of self-organized critical systems in general [2]. Following the well-known work of Bak, Tang and Wiesenfeld [3], many different types of sandpile models with different toppling rules have been studied [4]: deterministic and stochastic, with or without preferred direction, different instability criteria [5], or particle distribution rules [6], with fixed energy [7] etc.. However, a clear picture of the factors that determine different universality classes of critical behavior is yet to emerge [B].

A different paradigm for non-equilibrium critical phenomena has been directed percolation (DP) which is believed to describe active to absorbing state transition in a wide class of reaction-diffusion systems [9]. The activity of avalanches in sandpile models can grow, diffuse or die, and any stable configuration is an absorbing state. Thus, this should be in the universality class of DP with many absorbing states [10]. Several models of self-organized criticality which show critical exponents related to DP have been studied earlier [11]. However, these models do not involve any conserved field. The critical exponents of known models with conservation of sand are very different from those of DP, and this is presumably due to role of the local conservation of sand in the model. In [6], a model with conservation of particles showing DP exponents was studied, but this study was mainly numerical.

In this Letter we study several sandpile models with stochastic toppling rules, both directed and undirected. The grains are 'sticky' in our models in the sense that there is a nonzero probability that any grains arriving at a site during the avalanche process just get stuck there. We find that the distribution function of avalanches in these models has the same power law distribution as that of the critical DP clusters. Our theoretical arguments, supported by numerical simulations, show that generically these models belong to the DP universality class. The previously studied deterministic models [3, 12], and the stochastic toppling model introduced by Manna 13 are unstable to perturbations and flow to the DP fixed point.

The relation of sandpile models to DP was attempted earlier in 14] using sticky grains. However, in that paper, no bulk dissipation was present, and sand was dissipated only at the boundaries. The boundaries break the translational invariance of the steady state. The density becomes space dependent, and the density profile affects the statistics of avalanches. The critical exponents of avalanches are not those of critical DP clusters, but expressible in terms of DP exponents 15. Introducing bulk dissipation in this paper, we are able to get rid of these problems, and the relationship between the sandpile and DP problems becomes more transparent.

For simplicity, we start by defining the directed model on a $(1+1)$-dimensional square lattice. Various generalizations to higher dimensions, undirected case, and other toppling rules are straight forward, and briefly discussed at the end. The sites on an $L \times M$ torus are labelled by euclidean coordinates $(i, j)$ with $(i+j)$ even and $j$ increasing downwards. At each site $(i, j)$, there is a nonnegative integer $h_{i, j}$ to be called the height of the pile at that site. Initially all $h_{i, j}$ are zero. The system is driven by choosing a site at random and increasing the height at that site by one. If one or more particles are added to a site at time $t$ (from outside or from other sites), and its height becomes greater than 1 , then it is said to become unstable at time $t$.

Any site $(i, j)$ made unstable at time $t$ relaxes at the time $(t+1)$ stochastically: With probability $(1-p)$, it becomes stable without losing any grains. We say that the added particle(s) sticks to the existing grains. Otherwise (with probability $p$ ), the relaxation occurs by toppling in which the height at the site decreases by two, and the site becomes stable. We introduce bulk dissipation, by assuming that in each toppling, there is a small probability $\delta$ that both grains from the toppling are lost, other wise (with probability $1-\delta$ ), the two grains are transferred to the two downwards neighbors $(i \pm 1, j+1)$.

Note that there is a nonzero probability that a stable site can have arbitrarily large heights. We relax all the unstable sites by parallel dynamics. A site made unstable 
at time $t$ is relaxed in one step at time $t+1$, independent of whether it received one or more grains at the previous time step. Once a site has relaxed, it remains stable until perturbed again by new grains coming to the site. This relaxation process is repeated until all sites become stable, and then a new grain is added.

The model is specified by two parameters $p$ and $\delta$. The case $p=1, \delta=0$ is exactly soluble, and its critical exponents are known in all dimensions [12]. In this case, one has to introduce open boundary conditions to ensure the existence of a steady state. In two dimensions, the probability that adding a particle will cause an avalanche of s topplings varies as $s^{-\tau_{s}}$ for large $s$ with $\tau_{s}=4 / 3$. The probability that the duration of avalanche equals $T$ varies as $T^{-\tau_{t}}$ with $\tau_{t}=3 / 2$. The case $\delta=0, p$ arbitrary was studied earlier in [14 discussed in the introduction.

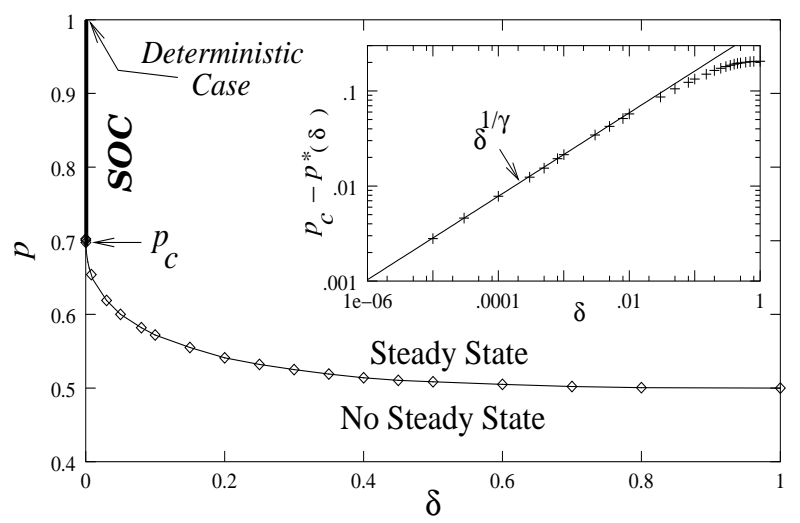

Fig. 1 The line $p=p^{*}(\delta)$ separating the steady state and no-steady-state regions in the $p-\delta$ plane. The inset shows a plot of $\left[p_{c}-p^{*}(\delta)\right]$ versus $\delta$. The straight line shows the theoretical slope $1 / \gamma$.

The probability distribution of different configurations in the steady state for general values of $p$ and $\delta$ have an interesting structure. Define a variable $g_{i, j}=h_{i, j}(\bmod 2)$. We group together different stable configurations $\left\{h_{i, j}\right\}$ corresponding to the same values of $\left\{g_{i, j}\right\}$. There are $2^{L M}$ such equivalence classes. On addition of a particle to a site, the $g$ variable at that site flips. A toppling leads to flipping of $g$ 's at the two downward sites. From detailed balance, it follows that in the steady state, each of the $2^{L M}$ equivalent classes occurs with equal probability [16.

If $p=1$, with $\delta$ arbitrary, the only allowed height values are 0 and 1 . In this case, we get a full characterization of the steady state. The $n$-point correlation functions satisfy linear equations, and exact solution of [12] can be generalized to arbitrary $\delta$. We omit the details here. The distribution of avalanche-sizes has an exponential decay for non-zero $\delta$.

As $p$ is decreased below 1 , heights greater than 1 appear with non-zero probability in the steady state. The mean height $\left\langle h_{i, j}\right\rangle$ increases as $p$ is decreased, and there exists a critical value $p^{*}(\delta)$ such that for $p \leq p^{*}(\delta)$, the height of the pile increases without bound, and there is no steady state (Fig. 1).

The absence of a steady state is obvious along the the line $p=0$, with $\delta$ arbitrary, as in this case, different sites cannot influence each other, and each added particle just sticks to the existing pile. A similar decoupling occurs along the line $\delta=1$, with $p$ arbitrary. In this case, the average particle loss per added grain is $2\left(1-c_{0}\right) p$, where $c_{0}$ is the density of sites with height 0 . In the steady state this must equal 1 . As $c_{0} \geq 0$, a steady state can exist only for $p>1 / 2$. Thus $p^{*}(\delta=1)=1 / 2$.

We now derive the equation for the boundary line $p=p^{*}(\delta)$. At the phase boundary, clearly $c_{0}=0$. In the growth of an avalanche in the steady state of the system, any site which receives at least one grain from its upward neighbors sends grains to downward neighbors with a probability $p(1-\delta)$. Thus the probability $\operatorname{Prob}(s)$ that an added grain will cause an avalanche in which at least $s$ sites transfer particles to downward neighbors is same as the probability $\operatorname{Prob}_{D P}(s \mid \tilde{p})$ of a cluster of at least $s$ sites in a directed site percolation process with concentration of active sites $=\tilde{p}$. A

$$
\operatorname{Prob}(s)=\operatorname{Prob}_{D P}(s \mid \tilde{p}=p(1-\delta)) .
$$

Note that Eq.(1) also holds in the entire 'no steady state' phase, where mean height continues to increase, but $\operatorname{Prob}(s)$ tends to a limiting distribution for large times.

By particle conservation, in the steady state, the average number of topplings in an avalanche must be equal to $1 /(2 \delta)$. Let $n_{D P}(\tilde{p})$ is the sum of the average number of occupied and perimeter sites in the cluster corresponding to a randomly picked site in the DP problem. Now, the equation for the phase boundary can be expressed in terms of the function $n_{D P}(\tilde{p})$ as

$$
n_{D P}\left((1-\delta) p^{*}(\delta)\right)=1 /(2 \delta) .
$$

For small $\delta$, the average size of clusters is large, and $\tilde{p}$ is near the critical probability $p_{c}$ for the directed site percolation on this lattice, the function $n_{D P}$ is known to vary as $\left(p_{c}-\tilde{p}\right)^{-\gamma}$. Substituting this in Eq.(2) we get

$$
p^{*}(\delta)=p_{c}-A \delta^{1 / \gamma}+\text { terms of higher order in } \delta,
$$

where $A$ is some constant, and $\gamma$ is the susceptibility exponent of the DP problem. In particular, we have $p^{*}(\delta=0)=p_{c}$. The inset in Fig. 1 shows a log-log plot of the numerically determined values of $p_{c}-p^{*}(\delta)$ plotted versus $\delta$. We get good agreement with Eq.(3) using the existing estimates $p_{c}=0.70548515$ and $\gamma=2.277730$ 17.

Since the average number of topplings in an avalanche in the steady state is $1 /(2 \delta)$, we will see self- organized criticality with long-ranged correlations only in the limit $\delta \rightarrow 0^{+}$, and $p_{c} \leq p \leq 1$, (marked with a heavy line in Fig. 1). For $\delta=0^{+}$and $1 \geq p>p_{c}$, in the steady state 
$c_{0}>0$. Each site is characterized by two probabilities, $p_{1}$ and $p_{2}$, which correspond to the probability that a site topples when one or two particles are added to it respectively. Clearly, $p_{1}=\left(1-c_{0}\right) p$ and $p_{2}=p$. The correlations between heights at different sites are small, and if we ignore them [18], the evolution of avalanches (Fig. 2) in the steady state is as in the Domany-Kinzel model of DP with parameters $\left(p_{1}, p_{2}\right)$ [19.

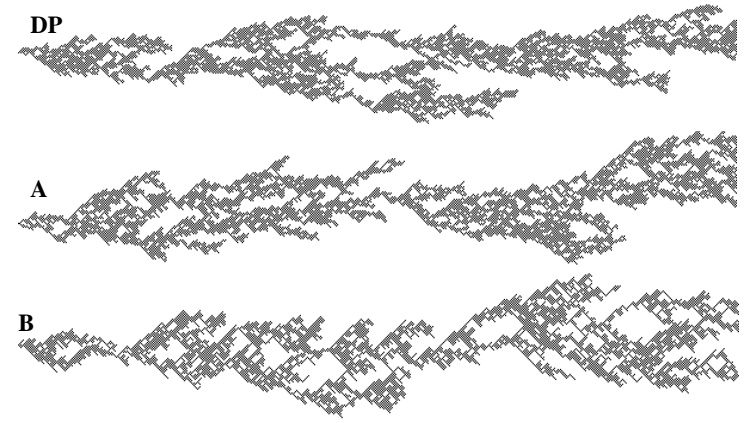

Fig. 2 Picture of a typical avalanche for the 2-d directed model $(\mathrm{A})$ and the time-evolution of the 1-d undirected model $(\mathrm{B})$ for $p=.873>p_{c}$ and $\delta=.0001$ are compared with the clusters at the critical line of the Domany-Kinzel model of DP with $p_{2}=p$.

Even if some short-range correlations are present, they should not modify critical behavior, which is expected to be same as in DP. In Fig. 3, we have compared the the probability distribution of avalanches with that of DP clusters. For $s \gg 1$, the latter is expected to satisfy the scaling form

$$
\operatorname{Prob}(s) \approx \frac{A}{s^{\tau-1}} f\left[s / s^{*}\right]
$$

where $A$ is some amplitudes, $\tau$ is a critical exponent for cluster size distribution in DP and the function $f(x)$ tends to a finite constant as $x$ tends to zero, and decreases exponentially with $x$ for large $x$. We assume that $s^{*}$ varies as $\delta^{-\phi}$. Using the constraint $\langle s\rangle \sim \delta^{-1}$, we get $\phi=1 /(2-\tau)$. Using the known numerical estimate $\tau=1.108$ in $d=1+1$ [17], we find a very good collapse when $s^{\tau-1} \operatorname{Prob}(s)$ is plotted versus $s \delta^{\phi}$ for two different values $p=p_{c}$ and $p=0.873$, and two values of $\delta=10^{-3}$ and $\delta=10^{-4}$. In the inset, the scaling function $f$ is compared with that for DP clusters. We get an excellent collapse, a strong evidence that the two functions are the same, and the correlations in heights in the steady state are irrelevant.

It is straightforward to extend the previous discussion to higher dimensions. Thus the avalanche exponents in the $(d+1)$-dimensional model are the same as the exponents of cluster size distribution in the $(d+1)$-dimensional DP at critical point. The upper critical dimension is $d=4$.

Consider now the undirected version of the problem on a $d$-dimensional hypercubical lattice. The rules are the same as before, except that on toppling, a particle is transferred to each of the $2 d$ neighbors of the toppling site. Clearly, in this case also, there is no steady state for small $p$. For $p=p^{*}(\delta)$, the mean height per site diverges. Then, any site which has one or more particles added to it, sends particles to its neighbors with probability $p(1-\delta)$. If we look at the space-time history of the evolution of the avalanche, we get a directed sitepercolation cluster on a $(d+1)$-dimensional body centered hypercubical (bch) lattice. The phase boundary in this undirected model is also the same as that of the directed model on the $(d+1)$-dimensional bch lattice.

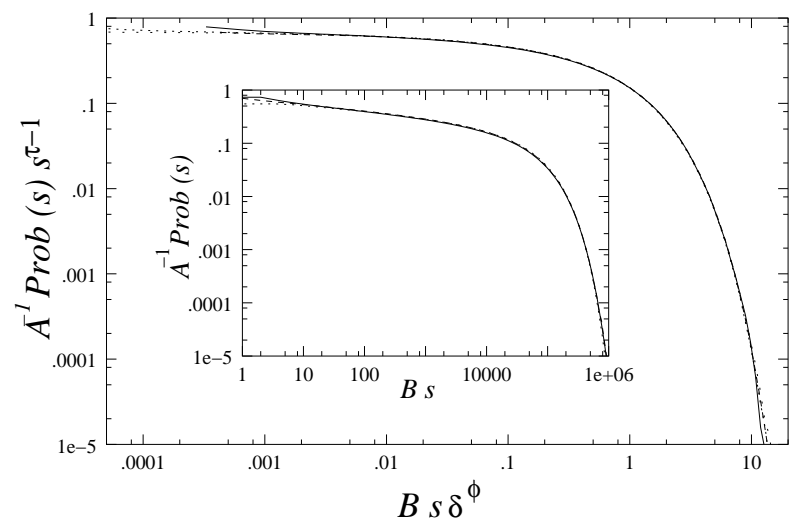

Fig. 3 Scaling collapse of $s^{\tau-1} \operatorname{Prob}(s)$ versus $s \delta^{\phi}$ for four different combinations of parameters $p, \delta$. The inset compares the scaling function for the directed and undirected models with $(p, \delta)=\left(0.873,10^{-4}\right)$ with that for $\mathrm{DP}$ at $p=0.7$.

For $p>p^{*}(\delta)$, the undirected model differs from the directed model in that the height of the pile at a site does not change between two topplings. This may give rise to possible long term memory effects. However, in our model this effect is rather small, as the probability of toppling depends on height only if the height is zero. Along the line $p=p^{*}(\delta)$, the memory affect is strictly absent, as the density of sites with zero height goes to zero. We find that even for $p$ as large as .873, the avalanches are qualitatively similar to DP (Fig. 2), and the distribution function is also indistinguishable from that of near-critical DP clusters (see inset of Fig. 3). There is a crossover from deterministic limit $(p=1, \delta=0)$ [3] to DP for $p \neq 1$.

Why does the conservation of particles not change the critical behavior away from DP in our problem? Consider a simple DP process of particles of type X, on a lattice with $n_{X}(\vec{r})$ particles of $\mathrm{X}$ at site $\vec{r}$. We now attach a register $n_{Y}(\vec{r})$ at each site $\vec{r}$, which decreases (increases) by 1 each time a particle $\mathrm{X}$ is created (destroyed) at $\vec{r}$. Then clearly, we have a local conservation of $n_{X}+n_{Y}$. Clearly, if the dynamics of the $\mathrm{X}$ particle is not affected by the book keeping, the process still belongs to the DP universality class. In our model, $n_{Y}$ is the height of the pile. It fluctuates about its mean value, but there is an influence of $n_{Y}$ on the dynamics of $\mathrm{X}$ particles as birth 
of $\mathrm{X}$ particles is not allowed if $n_{Y}$ is zero.

The phenomenological evolutions for the coarsegrained density fields $n_{X}(\vec{r})$ and $n_{Y}(\vec{r})$ in the conservative limit $\delta=0^{+}$may be written as 20]

$$
\begin{aligned}
& \partial_{t} n_{X}=\nabla^{2} n_{X}+a n_{X}-b n_{X}^{2}+c n_{X} g\left(n_{Y}\right)+\eta(\vec{r}, t) \\
& \partial_{t}\left(n_{X}+n_{Y}\right)=\nabla^{2} n_{X}
\end{aligned}
$$

where $\eta(\vec{r}, t)$ is a noise term, and $a, b$ 6and $c$ are phenomenological constants. The crucial term here is the coupling term $c n_{X} g\left(n_{Y}\right)$. Vespignani et al [20] chose $g\left(n_{Y}\right)$ proportional to $n_{Y}$. In our case, $n_{Y}$ has a threshold and the effect on $n_{X}$ saturates to a finite value even as $n_{Y}$ increases to infinity. The simplest choice of $g\left(n_{Y}\right)$ to model this is to choose $g\left(n_{Y}\right)=\theta\left(n_{Y}-h_{c}\right)$, where $\theta$ is the step function and $h_{c}$ is the threshold value. In naive power counting, this term has the same scaling as the linear term in $n_{X}$. We are not able to treat this analytically. But the results of our simulations strongly indicate that this perturbation does not change the critical exponents [21].

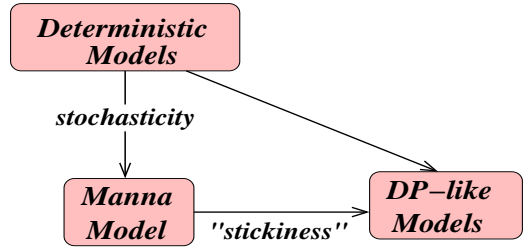

Fig. 4 A schematic flow diagram of renormalization group flows between different fixed points of sandpile models.

The DP fixed point is expected to be rather robust against perturbations. We have tested several variations of toppling rules in simulations. One can make the particle transfer process stochastic with each transferred particle going to a randomly chosen downward neighbor or one can allow multiple topplings at a site with each site toppling twice, thrice, etc., with decreasing probability, so long as the height is $>1$. With both multiple topplings and stochasticity in particle transfer, in the limit of no stickiness this becomes Manna model [13]. For sticky grains, in all these models, we get the DP behavior. The schematic renormalization group flows are shown in Fig. 4.

To summarize, we have studied several sandpile models which show DP-like behaviour. A feature, which is common in all these models is 'stickiness', i.e. with a nonzero probability a site can remain stable even with a height greater than the threshold. This behavior seems to be robust against perturbations, and is the generic behavior of sandpile models, both directed, and undirected.

We thank M. Barma, R. Dickman and A. Vespignani for their comments on the manuscript.
[1] H. M. Jaeger and S. R. Nagel, Science 255, 1523 (1992); Granular Matter, edited by A. Mehta (Springer, Heidelberg, 1994); H. J. Herrmann, Physica A 263, 51 (1999).

[2] Some recent reviews are H. J. Jensen, Self Organised Criticality (Cambridge University Press, England, 1998); D. L. Turcotte, Rep. Prog. Phys., 62, 1377 (1999); E. V. Ivashkevich and V. B. Priezzhev, Physica A 254, 97(1998); D. Dhar, Physica A 264, 1 (1999).

[3] P. Bak, C.Tang and K. Wiesenfeld, Phys. Rev. Lett. 59, 381 (1987); J. Phys. A38, 364(1988).

[4] L.P.Kadanoff, S. R. Nagel, L. Wu and S. M. Zhou, Phys. Rev. A 396524 (1989).

[5] S. S. Manna, Physica A 179, 249(1991).

[6] S. Maslov and Y-C. Zhang, Physica A 223, 1 (1996).

[7] A. Chessa, E. Marinari and A. Vespignani, Phys. Rev. Lett. 80, 4217 (1998).

[8] A. Ben-Hur and O. Biham, Phys. Rev. E 53, R1317 (1996).

[9] H. Hinrichsen, Advances in Phys., 49, 815(2000).

[10] M. A. Muñoz, R. Dickman, A. Vespignani and S. Zapperi, Phys. Rev. E 59, 6175(1999); R. Dickman, A. Vespignani and S. Zapperi, Phys. Rev. E 57, 5095(1998); R. Dickman, M. A. Munõz, A. Vespignani and S. Zapperi, Brazilian J. Phys., 30, 27 (2000).

[11] K. Sneppen, Physica A 221, 168(1995); P. Bak and K. Sneppen, Phys. Rev. Lett. 71, 4083 (1993); P. Grassberger and Y-C. Zhang, Physica A 224, 169(1996).

[12] D. Dhar and R. Ramaswamy, Phys. Rev. Lett. 63, 1659 (1989).

[13] S. S. Manna, J. Phys. A24, L363(1991).

[14] B. Tadic and D. Dhar, Phys. Rev. Lett. 79, 1519 (1997).

[15] For example, in $(1+1)$ dimensions, the exponent $\tau_{s}$ for the distribution of avalanches started at the top changes from the DP value 1.108 to a much bigger value 1.321 .

[16] A similar argument has been used earlier in A.B. Chhabra et al, Phys. Rev. E 473099 (1993).

[17] I. Jensen, J. Phys. A29, 7013(1996); ibid, J. Phys. A32, 5233(1999).

[18] Note that in the steady state, the heights are independently distributed along the three boundaries $p=0$, $p=1$ and $\delta=1$.

[19] E. Domany and W. Kinzel, Phys. Rev. Lett. 53, 311 (1984).

[20] A. Vespignani, R. Dickman, M. A. Muñoz and S. Zapperi, Phys. Rev. Lett. 81, 5676 (1998). ibid, Phys. Rev. E 62, 4564 (2000).

[21] A similar replacement from $\sqrt{n}$ to $\theta(n)$ in the amplitude of the noise term in evolution equation for the activity field makes the exact determination of critical exponents for the directed Manna model possible. See M. Paczuski and K. Bassler, Phys. Rev. E 62, 5347 (2000); M. Kloster, S. Maslov and C. Tang, Phys. Rev. E 63, 026111 (2001). 